Research Article

\title{
Neural Network Command Filtered Control of Fractional-Order Chaotic Systems
}

\author{
Hua Zhang (10 \\ Zhengzhou Preschool Education College, Zhengzhou 450099, China \\ Correspondence should be addressed to Hua Zhang; zhanghua27@163.com
}

Received 16 September 2021; Accepted 6 October 2021; Published 21 October 2021

Academic Editor: Wei Xiang

Copyright $\odot 2021$ Hua Zhang. This is an open access article distributed under the Creative Commons Attribution License, which permits unrestricted use, distribution, and reproduction in any medium, provided the original work is properly cited.

\begin{abstract}
An adaptive neural network (NN) backstepping control method based on command filtering is proposed for a class of fractionalorder chaotic systems (FOCSs) in this paper. In order to solve the problem of the item explosion in the classical backstepping method, a command filter method is adopted and the error compensation mechanism is introduced to overcome the shortcomings of the dynamic surface method. Moreover, an adaptive neural network method for unknown FOCSs is proposed. Compared with the existing control methods, the advantage of the proposed control method is that the design of the compensation signals eliminates the filtering errors, which makes the control effect of the actual system improve well. Finally, two examples are given to prove the effectiveness and potential of the proposed method.
\end{abstract}

\section{Introduction}

The fractional calculus equations are increasingly used to describe problems in optical and thermal systems, material and mechanical systems, signal processing, system identification, control, robotics, and other applications. The theory of fractional calculus has also been paid more and more attention by scholars at home and abroad, and especially, the fractional differential equations abstracted from practical problems have become the research focus of many mathematicians [1-3]. Bao and Cao [4] proved that fractionalorder differential equations can well simulate the dynamics of various chemical materials and special materials in practical applications. The traditional integer-order differential equation can be regarded as a special model of fractional-order differential equation. More information on fractional-order differential integration can be found in $[5,6]$. In recent years, many achievements have been made in the study of fractional-order calculus equations, which are used to study the problems of stability analysis and control of fractional-order strictly feedback nonlinear systems. For example, Li et al. [7] proposed a Lyapunov direct method for fractional-order nonlinear systems. Boroujeni and Momeni [8] proposed a fractional-order state observer for fractional- order nonlinear systems. Yang and Chen [9] presented a finite-time stabilization method for fractional-order switching systems. Adaptive control methods had been used in the control of fractional-order nonlinear systems $[5,6,10]$. Huang et al. [11] proposed a fuzzy feedback control method for uncertain fractional-order chaotic systems (FOCSs). Therefore, the research on the stability and control of FOCSs is becoming more and more popular.

The backstepping control was proposed for the first time to obtain asymptotic tracking and global stability of strictly feedback nonlinear systems [12]. The backstepping technique has always been a powerful tool for the design of controllers for nonlinear dynamic systems $[13,14]$. However, the classical backstepping control has two obvious disadvantages: one is the explosion of terms caused by the repeated derivation of virtual control signals [15-17], and the other is certain functions in the systems with uncertain parameters must be linear [18-20]. Li et al. [20] presented a fuzzy adaptive output feedback control method to improve the tracking effect of the systems. In addition, Shao-Cheng Tong et al. [21] proposed a backstepping method based on command filters to solve the problem of item explosion caused by repeated derivation of the virtual controller. Then, the command filtered backstepping control was extended to 
the adaptive case in [22]. In each step of the control design, the output of the command filter was used to approximate the differential coefficient of the virtual control signal, which eliminates the problem of item explosion. Note that the error compensation mechanism was designed to eliminate errors generated by the command filters [23]. However, the above works considered only the cases of systems with unknown parameters which are constant and systems without unknown parameters. In addition, the above research results only consider the case of integer-order systems, but the research results of the backstepping control method based on command filtering are relatively few for fractional-order strict feedback systems. In this paper, a backstepping control method based on command filtering is presented for a class of fractional-order strictly feedback nonlinear systems.

Based on the radial-basis-function neural network $(\mathrm{NN})$, an adaptive control method based on approximation theory was proposed to deal with nonlinear systems with uncertain parameters [24-28] or NN [29-31] approximation. The neural network control based on the backstepping control method of command filtering is used to solve nonlinear problems in uncertain systems. It can solve the nonlinear system which does not meet the matching condition and the certain functions are nonlinear [32-35]. For a class of FOCSs, an adaptive controller via the backstepping technology similar to [36, 37] was designed in [38]. However, because it is difficult to solve the fractional-order derivative of quadratic function, the adaptive backstepping control technology of integerorder nonlinear system cannot be directly applied to the fractional nonlinear system. For FOCSs, an adaptive backstepping control method was proposed in [39], and the stability of the controller was analyzed by the integerorder Lyapunov method. Therefore, for fractional-order nonlinear systems, how to design an adaptive backstepping controller via Lyapunov stability theory is an urgent problem to be solved.

According to the above discussion, an $\mathrm{NN}$ adaptive backstepping control method based on command filtering is proposed for FOCSs in this paper. The NN is used to deal with unknown functions in the system. The command filters are proposed to solve the problem of item explosion caused by repeated derivation of virtual controllers, and compensation signals are designed to eliminate the errors caused by the command filter. Based on NN approximation theory, an adaptive backstepping method based on command filtering is presented. The results show that this control method can adjust the tracking error to the small neighborhood of origin and ensure that all the closed-loop system signals have the limit boundedness of half blade uniformity.

The main advantages of this approach over current results can be summarized as follows:

(i) The command filtered adaptive NN backstepping control can solve two problems of classical backstepping of a class of fractional-order nonlinear systems and reduce the calculation burden.

(ii) An error compensation mechanism is introduced to overcome the shortcomings of the dynamic surface method, and the tracking error is controlled in the small neighborhood of zero.

The rest of this article is organized as follows: Section 2 is about the fuzzy logic system, fractional calculus description, and the fractional-order nonlinear systems. In Section 3, detailed controller design and stability analysis are given. In Section 4, simulation results are given to verify the effectiveness of the backstepping adaptive control method based on command filtering. Finally, Section 5 is the conclusion of this paper.

\section{Preliminaries and Problem Description}

2.1. Preliminaries of Fractional-Order Calculus. In recent several decades, the relatively common two kinds of fractional-order calculus are Riemann-Liouville (RL) fractionalorder calculus and Caputo fractional-order calculus. The nice property of the Caputo fractional-order differential equation is that its integral value at zero makes sense. Therefore, this paper will adopt the definition of Caputo fractional-order calculus.

The fractional-order calculus operators can be regarded as a broader concept of integral calculus operators.

Thus, the definition of the fractional-order integral can be expressed as [1]

$$
{ }_{0}^{C} I_{t}^{\beta} x(t)=\frac{1}{\Gamma(\beta)} \int_{0}^{t} \frac{x(\zeta)}{(t-\zeta)^{1-\beta}} \mathrm{d} \zeta,
$$

where $\Gamma(\cdot)$ is the gamma function and $\Gamma(s)=\int_{0}^{+\infty} t^{s-1} e^{-t} \mathrm{~d} t$. Consequently, the fractional-order derivative operator is represented by [1]

$$
{ }_{0}^{C} D_{t}^{\beta} x(t)=\frac{1}{\Gamma(n-\beta)} \int_{0}^{t} \frac{x^{(n)}(\zeta)}{(t-\zeta)^{\beta+1-n}} \mathrm{~d} \zeta .
$$

The Laplace transform of (2) can be expressed as

$$
\mathrm{L}\left[{ }_{0}^{C} D_{t}^{\beta} x(t)\right]=s^{\beta} X(s)-\sum_{k=0}^{n-1} s^{\beta-k-1} x^{(k)}(0),
$$

where $X(s)$ is the Laplace transform of $X(t)$.

In the following sections, we examine only the case of $\beta \in(0,1)$.

Definition 1 (see [1]). The Mittag-Leffler function can be expressed as

$$
E_{\beta, \gamma}(z)=\sum_{k=0}^{+\infty} \frac{z^{k}}{\Gamma(k \beta+\gamma)},
$$

with $\beta, \gamma>0$, and $z \in C$. The Laplace transform of (5) can be described as

$$
\mathrm{L}\left\{t^{\gamma-1} E_{\beta, \gamma}\left(-a t^{\beta}\right)\right\}=\frac{s^{\beta-\gamma}}{s^{\beta}+a} .
$$

Lemma 1 (see [1]). If there is a complex number $\gamma$ and two real numbers $\beta(0<\beta<1)$ and $₫$, where $₫$ satisfies the following condition: 


$$
\frac{\pi \beta}{2}<\omega<\min \{\pi, \pi \beta\}
$$

then, the following formula is true for all integers $n \geq 1$ :

$$
E_{\beta, \gamma}(z)=\sum_{k=0}^{n} \frac{1}{\Gamma(\gamma-\beta j) z^{j}}+o\left(\frac{1}{|z|^{n+1}}\right),
$$

when $|z| \longrightarrow \infty$ and $\omega \leq|\arg (z)| \leq n$.

Lemma 2 (see [1]). If $\beta(0<\beta<2)$ and $\gamma$ are two arbitrary real numbers, and there exists a positive constant $\varrho$ satisfying the following condition:

$$
\left(\frac{v \pi}{2}\right)<\varrho \leq \min \{\pi, \beta \pi\},
$$

then the following inequality holds:

$$
\left|E_{\beta, \gamma}(z)\right| \leq \frac{C}{1+|z|},
$$

where $C>0, \varrho \leq|\arg (z)| \leq \pi$, and $|z| \geq 0$.

Definition 2 (see [7]). Suppose $w:[0, c) \longrightarrow \mathscr{I}$ is a continuous function. If the continuous function is strictly increasing and $w(0)=0$, then it is said to be class $\mathrm{K}$.

Lemma 3 (see [7]). If an equilibrium point of fractionalorder nonlinear system $\mathscr{D}_{t}^{\beta} y(t)=g(t, t(x))$ is origin, which $g: \mathscr{I} \times \Omega \longrightarrow \mathscr{R}$ is Lipschitz continuous. There exist a Lyapunov function $V(t, y(t))$ and class $K$ functions $w_{i}(i=$ $1,2,3)$ to satisfy

$$
\begin{aligned}
w_{1}(\|y(t)\|) & \leq V(t, y(t)) \leq w_{2}(\|y(t)\|), \\
\mathscr{D}_{t}^{\beta} V(t, y(t)) & \leq w_{3}(\|y(t)\|) .
\end{aligned}
$$

Then, $\mathscr{D}_{t}^{\beta} y(t)=g(t, t(x))$ is asymptotically stable; i.e., $\lim _{t \rightarrow \infty} y(t)=0$.

Lemma 4 (see [2]). Assume that $y(t) \in \mathscr{C}^{1}\left(\left[t_{0}, \infty\right], \mathscr{R}^{n}\right)$, then

$$
\frac{1}{2} \mathscr{D}_{t}^{\beta}\left(y^{T}(t) y(t)\right) \leq y^{T}(t) \mathscr{D}_{t}^{\beta} y(t), \quad(\forall t \in \mathscr{I}) .
$$

2.2. Preliminaries of Radial-Basis-Function NN. Let $h(\chi(t))$ be a continuous unknown nonlinear function defined over a compact set $\Omega$. Then, there exists a radial-basis-function $\mathrm{NN}$ to appropriate the unknown nonlinear function $h(\chi(t))$ as follows:

$$
\widehat{h}(\boldsymbol{\chi}(t))=\boldsymbol{\theta}^{T} \psi(\boldsymbol{\chi}(t)),
$$

where $\hat{h}: \mathscr{R}^{n} \longrightarrow \mathscr{R}(n \in N)$ is a continuous mapping, $\chi(t)=\left[\chi_{1}(t), \chi_{2}(t), \ldots, \chi_{n}(t)\right]^{T}$ is the $\mathrm{NN}$ input vector, $t \geq 0$, $\chi_{i}(t) \in \mathscr{C}^{1}[\mathscr{I}, \Omega] \quad(i=1,2, \ldots, n), \quad \theta=\left[\theta_{1}, \theta_{2}, \ldots, \theta_{N}\right]^{T} \in$ $\mathscr{R}^{N} \quad(N>1)$ is the weight vector, $\psi(\chi(t))=\left[\psi_{1}\right.$ $\left.(\chi(t)), \psi_{2}(\chi(t)), \ldots, \psi_{N}(\chi(t))\right]^{T} \in \mathscr{R}^{N}$ is a regressor, and $N$ is the number of NN nodes. The regression variable $\psi_{1}(\chi(t))$ selected in this paper is the Gaussian radial-basis-function

$$
\psi_{1}(\chi(t))=\exp \left(\frac{-\left\|x-\delta_{i}\right\|^{2}}{2 \iota_{i}^{2}}\right)
$$

where $\quad 1=1,2, \ldots, \mathrm{N}, \quad \delta_{1}=\left[\delta_{11}, \delta_{21}, \ldots, \delta_{n 1}\right]^{T} \in R^{n}, \quad \delta_{\mathscr{g}_{1}}=$ $(\mathscr{J}=1,2, \ldots, n)$ represent the centers of the Gaussian function, and $\iota_{1} \in R^{+}$represent the widths of the Gaussian function.

Therefore, according to the above notation, the optimal estimate of $h(\chi(t))$ can be expressed as

$$
h(\chi(t))=\boldsymbol{\theta}^{* T} \boldsymbol{\psi}(\boldsymbol{\chi}(t))+\epsilon(\boldsymbol{\chi}(t)),
$$

in which $\epsilon(\chi(t))=\left[\epsilon_{1}\left(\chi_{1}(t)\right), \epsilon_{2}\left(\chi_{2}(t)\right), \ldots, \epsilon_{N}\left(\chi_{N}(t)\right)\right]$ and $\theta^{*}$ is the optimal constant weight vector and satisfies the following condition:

$$
\boldsymbol{\theta}^{*}(t)=\arg \min _{\boldsymbol{\theta}}[\sup \chi(t)|h(\chi(t))-\widehat{h}(\boldsymbol{\chi}(t))|]
$$

Let

$$
\widetilde{\boldsymbol{\theta}}=\boldsymbol{\theta}-\boldsymbol{\theta}^{*},
$$

in which $\widetilde{\theta}$ is the parameter estimation error. According to the properties of the radial-basis-function $\mathrm{NN}$, it is assumed that the optimal approximation error remains bounded. For any $\bar{\epsilon}$, there exists a sufficient number of NN nodes such that

$$
\left|\epsilon_{1}\left(\chi_{1}(t)\right)\right| \leq \bar{\epsilon}_{1}
$$

where $\bar{\epsilon}_{1}$ is a known positive constant and $1=1,2, \ldots, \mathrm{N}$.

Then, one can obtain

$$
\begin{aligned}
\widehat{h}_{1} & \left(\chi_{1}(t), \theta_{1}\right)-h_{1}\left(\chi_{1}(t)\right) \\
& =\widehat{h}_{1}\left(\chi_{1}(t), \theta_{1}\right)-\widehat{h}_{1}\left(\chi_{1}(t), \theta_{1}^{*}\right)+\widehat{h}_{1}\left(\chi_{1}(t), \theta_{1}^{*}\right)-h_{1}\left(\chi_{1}(t)\right) \\
& =\theta_{1}^{T} \psi_{1}\left(\chi_{1}(t)\right)-\theta_{1}^{* T} \psi_{1}\left(\chi_{1}(t)\right)+\epsilon_{1}\left(\chi_{1}(t)\right) \\
& =\widetilde{\theta}_{1}^{T} \psi_{1}\left(\chi_{1}(t)\right)+\epsilon_{1}\left(\chi_{1}(t)\right),
\end{aligned}
$$

in which $1=1,2, \ldots, \mathrm{N}$.

2.3. FOCSs. Consider the following FOCSs:

$$
\left\{\begin{array}{l}
\mathscr{D}_{t}^{\beta} x_{1}(t)=x_{2}(t)+f_{1}\left(\overline{x_{1}}\right) \\
\mathscr{D}_{t}^{\beta} x_{i}(t)=x_{i+1}(t)+f_{i}\left(\overline{x_{i}}\right) \\
\mathscr{D}_{t}^{\beta} x_{n}(t)=f_{n}(x(t))+d(t)+u(t) \\
y(t)=x_{1}(t)
\end{array}\right.
$$

where $\beta(0<\beta<1)$ is the system commensurate order, $\mathbf{x}(t)=$ $\left[{ }_{1}(t), x_{2}(t), \ldots, x_{n}(t)\right]^{T} \in \mathscr{R}^{n}$ is the state vector of the system, $\bar{x}(t)=\left[x_{1}(t), x_{2}(t), \ldots, x_{i}(t)\right]^{T} \in \mathscr{R}^{i}, f_{i}\left(\bar{x}_{i}\right) \in \mathscr{R}$ $(i=1,2, \ldots, n)$ are unknown smooth nonlinear functions, $d(t) \in \mathscr{R}$ is an unknown external disturbance, and $u(t) \in \mathscr{R}$ and $y(t) \in \mathscr{R}$ are control input and control output of the fractional-order strictly feedback nonlinear systems. 
Assumption 1. For the FOCSs, if $d(t)$ is bounded and unknown, then there exists an unknown constant $\bar{d}(\bar{d}>0)$ such that $|d(t)| \leq \bar{d}$ for all $t \geq 0$.

\section{Adaptive NN Backstepping Control Design}

Let

$$
\begin{aligned}
& s_{1}(t)=x_{1}(t)-x_{d}, \\
& s_{i}(t)=x_{i}(t)-x_{i, c},
\end{aligned}
$$

in which $x_{d}$ is a known reference signal and $x_{i, c}$ $(i=2,3, \ldots, n)$ are the command filters.

The command filtering mechanism is defined as follows:

$$
\mathscr{D}_{t}^{\beta} x_{i+1, c}(t)=-\kappa_{i}\left(x_{i+1, c}(t)-\alpha_{i}(t)\right),
$$

where $\kappa_{i}$ is a positive constant and $\alpha_{i}(t)$ and $x_{i+1, c}(t)$ $(i=1,2, \ldots, n-1)$ are the input signal and the output signal in the command filtering mechanism, respectively.

It is worth noting that command filtering produces errors that add to the burden of getting better tracking results. To solve this problem, an error compensation mechanism is presented to overcome the errors $\left(x_{i+1, c}-\alpha_{i}\right)$ generated during the filtering process.

Then, the error compensation mechanism is defined as

$$
\begin{aligned}
& \mathscr{D}_{t}^{\beta} \lambda_{1}(t)=-k_{11} \lambda_{1}(t)+\lambda_{2}+x_{2, c}-\alpha_{1}, \\
& \mathscr{D}_{t}^{\beta} \lambda_{i}(t)=-k_{1 i} \lambda_{i}(t)-\lambda_{i-1}+\lambda_{i+1}+x_{i+1, c}-\alpha_{i}, \\
& \mathscr{D}_{t}^{\beta} \lambda_{n}(t)=-k_{1 n} \lambda_{n}(t)-\lambda_{n-1},
\end{aligned}
$$

where $k_{1 i}>0$ are design parameters and $\lambda_{i}$ $(i=1,2, \ldots, n-1)$ are the error compensating signals.

The compensated tracking error signals $e_{i}(t)$ are designed as

$$
e_{i}(t)=s_{i}(t)-\lambda_{i}(t)
$$

in which $i=1,2, \ldots, n$.

Our goal is to design the control input $u$ of the nonlinear systems such that the control output $y(t)$ of the system to track $x_{d}$ and the compensated tracking error of the nonlinear systems $e_{1}(t)$ eventually converge to a sufficiently small region of origin.

Then, the virtual control functions $\alpha_{i}(i=1,2, \ldots, n)$ are constructed as

$$
\begin{aligned}
& \alpha_{1}=-\theta_{1}^{T} \psi_{1}\left(\bar{x}_{1}(t)\right)-k_{11} s_{1}(t)-k_{21} \operatorname{sign}\left(e_{1}(t)\right)+\mathscr{D}_{t}^{\beta} x_{d}(t), \\
& \alpha_{2}=-\theta_{2}^{T} \psi_{2}\left(\bar{x}_{2}(t)\right)-k_{12} s_{2}(t)-k_{22} \operatorname{sign}\left(e_{2}(t)\right)-s_{1}(t)+\mathscr{D}_{t}^{\beta} x_{2, c}(t), \\
& \alpha_{i}=-\theta_{i}^{T} \psi_{i}\left(\bar{x}_{i}(t)\right)-k_{1 i} s_{i}(t)-k_{2 i} \operatorname{sign}\left(e_{i}(t)\right)-s_{i-1}(t)+\mathscr{D}_{t}^{\beta} x_{i, c}(t),
\end{aligned}
$$

where $k_{2 i}>\bar{\epsilon}_{i}(i=1,2, \ldots, n-1)$ are design parameters and

The controller $u(t)$ is designed as follows: $\bar{\epsilon}_{i}$ will be determined later.

$$
u(t)=-\theta_{n}^{T} \psi_{n}\left(x_{n}(t)\right)-k_{1 n} s_{n}(t)-s_{n-1}(t)-\left(k_{2 n}+\hat{\bar{d}}(t)\right) \operatorname{sign}\left(e_{n}(t)\right)+\mathscr{D}_{t}^{\beta} x_{n, c}(t),
$$

in which $\hat{\bar{d}}(t)$ is the estimation of $\bar{d}, k_{2 n}>\bar{\epsilon}_{n}$ is a design parameter, and $\bar{\epsilon}_{n}$ will be determined later.

Then, the backstepping control algorithms of the fractional-order nonlinear system are shown as follows.
Step 1. Consider the first Lyapunov function $v_{1}(x)$ as follows:

$$
v_{1}(t)=\frac{1}{2} e_{1}^{2}(t)
$$


According to Lemma 4 , differentiating $v_{1}(x)$ can gain

$$
\begin{aligned}
\mathscr{D}_{t}^{\beta} v_{1}(t) \leq & e_{1}(t) \mathscr{D}_{t}^{\beta} e_{1}(t) \\
= & e_{1}(t)\left(D_{t}^{\beta} x_{1}(t)-\mathscr{D}_{t}^{\beta} x_{d}(t)-\mathscr{D}_{t}^{\beta} \lambda_{1}(t)\right) \\
= & e_{1}(t)\left[x_{2}(t)+f_{1}\left(\bar{x}_{1}(t)\right)-\mathscr{D}_{t}^{\beta} x_{d}(t)-\mathscr{D}_{t}^{\beta} \lambda_{1}(t)\right] \\
= & e_{1}(t)\left[s_{2}(t)+x_{2, c}(t)+f_{1}\left(\bar{x}_{1}(t)\right)-\widehat{f}_{1}\left(\bar{x}_{1}\left(t, \theta_{1}^{*}\right)\right)+\widehat{f}_{1}\left(\bar{x}_{1}\left(t, \theta_{1}^{*}\right)\right)\right. \\
& -\mathscr{D}_{t}^{\beta} x_{d}(t)+k_{11} \lambda_{1}(t)-\lambda_{2}(t)-x_{2, c}(t)+\beta_{1}(t) \\
= & e_{1}(t)\left[e_{2}(t)-\widetilde{\theta}_{1}^{T} \psi_{1}\left(\bar{x}_{1}(t)\right)-\epsilon_{1}\left(\bar{x}_{1}(t)\right)+\theta_{1}^{T} \psi_{1}\left(\bar{x}_{1}(t)\right)-\mathscr{D}_{t}^{\beta} x_{d}(t)+k_{11} \lambda_{1}(t)+\alpha_{1}(t),\right.
\end{aligned}
$$

in which $\epsilon_{1}\left(\bar{x}_{1}(t)\right)=\widehat{f}_{1}\left(\bar{x}_{1}\left(t, \theta^{*}\right)\right)-f_{1}\left(\bar{x}_{1}(t)\right)$ and $\widetilde{\theta}_{1}=$ $\theta_{1}-\theta_{1}^{*}$.
Substituting (25) into (30), we can obtain

$$
\begin{aligned}
\mathscr{D}_{t}^{\beta} v_{1}(t) & \leq e_{1}(t)\left[e_{2}(t)-k_{11} e_{1}(t)-\tilde{\theta}_{1}^{T} \psi_{1}\left(\bar{x}_{1}(t)\right)-\epsilon_{1}\left(\bar{x}_{1}(t)\right)-k_{21} \operatorname{sign}\left(e_{1}(t)\right)\right] \\
& \leq e_{1}(t) e_{2}(t)-k_{11} e_{1}^{2}(t)-e_{1}(t) \widetilde{\theta}_{1}^{T} \psi_{1}\left(\bar{x}_{1}(t)\right)+\bar{\epsilon}_{1}\left|e_{1}(t)\right|-k_{21}\left|e_{1}(t)\right| \\
& \leq e_{1}(t) e_{2}(t)-k_{11} e_{1}^{2}(t)-e_{1}(t) \widetilde{\theta}_{1}^{T} \psi_{1}\left(\bar{x}_{1}(t)\right),
\end{aligned}
$$

where $k_{11}>0$ and $k_{21}>\bar{\epsilon}_{1}$ are design parameters.

Choose the Lyapunov function $V_{1}(x)$ as follows:

$$
V_{1}(t)=v_{1}(t)+\frac{1}{2 \rho_{1}} \tilde{\theta}_{1}^{T}(t) \tilde{\theta}_{1}(t) .
$$

The design of adaptive law is as follows:

$$
\mathscr{D}_{t}^{\beta} \theta_{1}=\rho_{1} e_{1}(t) \psi_{1}\left(\bar{x}_{1}(t)\right)-\gamma_{1} \theta_{1},
$$

where $\rho_{1}$ and $\gamma_{1}$ are positive design parameters.

Because $\theta_{1}^{*}$ is a constant, then we have

$$
\mathscr{D}_{t}^{\beta} \tilde{\theta}_{1}=\mathscr{D}_{t}^{\beta} \theta_{1} \text {. }
$$

Then, according to Lemma 4, we can have

$$
\mathscr{D}_{t}^{\beta} V_{1}(t) \leq \mathscr{D}_{t}^{\beta} v_{1}(t)+\frac{1}{\rho_{1}} \widetilde{\theta}_{1}^{T} \mathscr{D}_{t}^{\beta} \widetilde{\theta}_{1} .
$$

Substituting formulas (31) and (33) into the above inequality, the following can be obtained:

$$
\begin{aligned}
\mathscr{D}_{t}^{\beta} V_{1}(t) & \leq-k_{11} e_{1}^{2}(t)+e_{1}(t) e_{2}(t)-\frac{1}{\rho_{1}} \widetilde{\theta}_{1}^{T} \theta_{1} \\
& \leq-k_{11} e_{1}^{2}(t)+e_{1}(t) e_{2}(t)-\frac{1}{\rho_{1}} \widetilde{\theta}_{1}^{T} \widetilde{\theta}_{1}-\frac{1}{\rho_{1}} \tilde{\theta}_{1}^{T} \theta_{1}^{*} .
\end{aligned}
$$

According to Young's inequality, then we can obtain

$$
-\frac{1}{\rho_{1}} \widetilde{\theta}_{1}^{T} \theta_{1}^{*} \leq \frac{1}{2 \rho_{1}} \theta_{1}^{* T} \theta_{1}^{*}+\frac{1}{2 \rho_{1}} \widetilde{\theta}_{1}^{T} \widetilde{\theta}_{1} .
$$

According to formulas (36) and (37), it can be obtained

$$
\begin{aligned}
\mathscr{D}_{t}^{\beta} V_{1}(t) & \leq-k_{11} e_{1}^{2}(t)+e_{1}(t) e_{2}(t)-\frac{1}{2 \rho_{1}} \widetilde{\theta}_{1}^{T} \widetilde{\theta}_{1}+\frac{1}{2 \rho_{1}} \theta_{1}^{* T} \theta_{1}^{*} \\
& \leq-k_{1} V_{1}(t)+e_{1}(t) e_{2}(t)+\Theta_{1},
\end{aligned}
$$

in which $k_{1}=\min \left\{2 k_{11}, \gamma_{1}\right\}$ and $\Theta_{1}=\left(\gamma_{1} / 2 \rho_{1}\right) \theta_{1}^{* T} \theta_{1}^{*}$ are two positive constants.

Step 2. Consider the second Lyapunov function $v_{2}(x)$ as follows:

$$
v_{2}(t)=\frac{1}{2} e_{2}^{2}(t)
$$

According to Lemma 4, differentiating $v_{2}(x)$ can gain 


$$
\begin{aligned}
\mathscr{D}_{t}^{\beta} v_{2}(t) \leq e_{2}(t) \mathscr{D}_{t}^{\beta} e_{2}(t) \\
=e_{2}(t)\left(D_{t}^{\beta} x_{2}(t)-\mathscr{D}_{t}^{\beta} x_{2, c}(t)-\mathscr{D}_{t}^{\beta} \lambda_{2}(t)\right) \\
=e_{2}(t)\left[x_{3}(t)+f_{2} \bar{x}_{2}(t)-\mathscr{D}_{t}^{\beta} x_{2, c}(t)-\mathscr{D}_{t}^{\beta} \lambda_{2}(t)\right] \\
=e_{2}(t) s_{3}(t)+x_{3, c}(t)+f_{2}\left(\bar{x}_{2}(t)\right)-\hat{f}_{2}\left(\bar{x}_{2}\left(t, \theta_{2}^{*}\right)\right)+\widehat{f}_{2}\left(\bar{x}_{2}\left(t, \theta_{2}^{*}\right)\right) \\
\quad-\mathscr{D}_{t}^{\beta} x_{2, c}(t)+k_{12} \lambda_{2}+\lambda_{1}(t)-\lambda_{3}(t)-x_{3, c}(t)+\alpha_{2}(t) \\
=e_{2}(t) e_{3}(t)-\tilde{\theta}_{2}^{T} \psi_{2}\left(\bar{x}_{2}(t)\right)-\epsilon_{2}\left(\bar{x}_{2}(t)\right)+\theta_{2}^{T} \psi_{2}\left(\bar{x}_{2}(t)\right)-\mathscr{D}_{t}^{\beta} x_{2, c}(t) \\
\quad+k_{12} \lambda_{2}+\lambda_{1}(t)+\alpha_{2}(t),
\end{aligned}
$$

in which $\epsilon_{2}\left(\bar{x}_{2}(t)\right)=\widehat{f}_{2}\left(\bar{x}_{2}\left(t, \theta^{*}\right)\right)-f_{2}\left(\bar{x}_{2}(t)\right)$ and $\widetilde{\theta}_{2}=$ $\theta_{2}-\theta_{2}^{*}$.

$$
\begin{aligned}
& \mathscr{D}_{t}^{\beta} v_{2}(t) \leq e_{2}(t)\left[e_{3}(t)-k_{12} e_{2}(t)-\widetilde{\theta}_{2}^{T} \psi_{2}\left(\bar{x}_{2}(t)\right)-\epsilon_{2}\left(\bar{x}_{2}(t)\right)-k_{22} \operatorname{sign}\left(e_{2}(t)\right)-e(t)\right] \\
& \leq e_{2}(t) e_{3}(t)-k_{12} e_{2}^{2}(t)-e_{2}(t) \widetilde{\theta}_{2}^{T} \psi_{2}\left(\bar{x}_{2}(t)\right)+\bar{\epsilon}_{2}\left|e_{2}(t)\right| \\
& \quad-k_{22}\left|e_{2}(t)\right|-e_{1}(t) e_{2}(t) \\
& \leq-k_{12} e_{2}^{2}+e_{2}(t) e_{3}(t)-e_{2}(t) \tilde{\theta}_{2}^{T} \psi_{2}\left(\bar{x}_{2}(t)\right)-e_{1}(t) e_{2}(t),
\end{aligned}
$$

where $k_{12}>0$ and $k_{22}>\bar{\varepsilon}_{2}$ are design parameters.

Choose the Lyapunov function $V_{2}(x)$ as follows:

$$
V_{2}(t)=V_{1}(t)+v_{2}(t)+\frac{1}{2 \rho_{2}} \tilde{\theta}_{2}^{T}(t) \widetilde{\theta}_{2}(t)
$$

The design of adaptive law is as follows:

$$
\mathscr{D}_{t}^{\beta} \theta_{2}=\rho_{2} e_{2}(t) \psi_{2}\left(\bar{x}_{2}(t)\right)-\gamma_{2} \theta_{2} \text {, }
$$

where $\rho_{2}$ and $\gamma_{2}$ are positive design parameters.

Because $\theta_{2}^{*}$ is a constant, then we have

$$
\mathscr{D}_{t}^{\beta} \widetilde{\theta}_{2}=\mathscr{D}_{t}^{\beta} \theta_{2} \text {. }
$$

Then, according to Lemma 4 and formulas (42) and (44), we can have

$$
\mathscr{D}_{t}^{\beta} V_{2}(t) \leq-k V_{1}(t)+e_{1}(t) e_{2}(t)+\Theta_{1}+\mathscr{D}_{t}^{\beta} v_{2}(t)+\frac{1}{\rho_{2}} \widetilde{\theta}_{2}^{T} \mathscr{D}_{t}^{\beta} \widetilde{\theta}_{2}
$$

According to formulas (45) and (46), the above equation can be reduced to

$$
\begin{aligned}
\mathscr{D}_{t}^{\beta} V_{2}(t) & \leq-k_{1} V_{1}(t)+\Theta_{1}-k_{12} e_{2}^{2}(t)+e_{2}(t) e_{3}(t)-\frac{1}{2 \rho_{2}} \widetilde{\theta}_{2}^{T} \widetilde{\theta}_{2}+\frac{1}{2 \rho_{2}} \theta_{2}^{* T} \theta_{2}^{*} \\
& \leq-k_{2} V_{2}(t)+e_{2}(t) e_{3}(t)+\Theta_{2}
\end{aligned}
$$

in which $k_{2}=\min \left\{k_{1}, 2 k_{12}, \gamma_{2}\right\}$ and $\Theta_{2}=\Theta_{1}+\left(\gamma_{2} / \quad\right.$ Step 3. $i(i=3,4, \ldots, n-1)$. $\left.2 \rho_{2}\right) \theta_{2}^{* T} \theta_{2}^{*}$ are two positive constants.

Consider the $i$ th Lyapunov function $v_{i}(x)$ as follows: 


$$
v_{i}(t)=\frac{1}{2} e_{i}^{2}(t)
$$

$$
\begin{aligned}
\mathscr{D}_{t}^{\beta} v_{i}(t) \leq e_{i}(t) \mathscr{D}_{t}^{\beta} e_{i}(t) \\
=e_{i}(t)\left(D_{t}^{\beta} x_{i}(t)-\mathscr{D}_{t}^{\beta} x_{i, c}(t)-\mathscr{D}_{t}^{\beta} \lambda_{i}(t)\right) \\
=e_{i}(t)\left[x_{i+1}(t)+f_{i}\left(\bar{x}_{i}(t)\right)-\mathscr{D}_{t}^{\beta} x_{i, c}(t)-\mathscr{D}_{t}^{\beta} \lambda_{i}(t)\right] \\
=e_{i}(t) s_{i+1}(t)+x_{i+1, c}(t)+f_{i}\left(\bar{x}_{i}(t)\right)-\widehat{f}_{i}\left(\bar{x}_{i}\left(t, \theta_{i}^{*}\right)\right)+\widehat{f}_{i}\left(\bar{x}_{i}\left(t, \theta_{i}^{*}\right)\right) \\
\quad-\mathscr{D}_{t}^{\beta} x_{i, c}(t)+k_{1 i} \lambda_{i}+\lambda_{i-1}(t)-\lambda_{i+1}(t)-x_{i+1, c}(t)+\alpha_{i}(t) \\
=e_{i}(t) e_{i+1}(t)-\tilde{\theta}_{i}^{T} \psi_{i}\left(\bar{x}_{i}(t)\right)-\epsilon_{i}\left(\bar{x}_{i}(t)\right)+\theta_{i}^{T} \psi_{i}\left(\bar{x}_{i}(t)\right) \\
\quad-\mathscr{D}_{t}^{\beta} x_{i, c}(t)+k_{1 i} \lambda_{i}+\lambda_{i-1}(t)+\alpha_{i}(t)
\end{aligned}
$$

in which $\epsilon_{i}\left(\bar{x}_{i}(t)\right)=\widehat{f}_{i}\left(\bar{x}_{i}\left(t, \theta^{*}\right)\right)-f_{i}\left(\bar{x}_{i}(t)\right) \quad$ and $\quad$ Substituting (27) into (49), we can obtain $\widetilde{\theta}_{i}=\theta_{i}-\theta_{i}^{*}$.

$$
\begin{aligned}
& \mathscr{D}_{t}^{\beta} v_{i}(t) \leq e_{i}(t)\left[e_{i+1}(t)-k_{1 i} e_{i}(t)-\tilde{\theta}_{i}^{T} \psi_{i}\left(\bar{x}_{i}(t)\right)-\epsilon_{i}\left(\bar{x}_{i}(t)\right)-k_{2 i} \operatorname{sign}\left(e_{i}(t)\right)-e_{i-1}(t)\right] \\
& \leq e_{i}(t) e_{i+1}(t)-k_{1 i} e_{i}^{2}(t)-e_{i}(t) \tilde{\theta}_{i}^{T} \psi_{i}\left(\bar{x}_{i}(t)\right)+\bar{\epsilon}_{i}\left|e_{i}(t)\right|-k_{2 i}\left|e_{i}(t)\right|-e_{i-1}(t) e_{i}(t) \\
& \leq e_{i}(t) e_{i+1}(t)-k_{1 i} e_{i}^{2}-e_{i}(t) \tilde{\theta}_{i}^{T} \psi_{i}\left(\bar{x}_{i}(t)\right)-e_{i-1}(t) e_{i}(t),
\end{aligned}
$$

where $k_{1 i}>0$ and $k_{2 i}>\bar{\epsilon}_{i}$ are design parameters.

Choose the Lyapunov function $V_{i}(x)$ as follows:

$$
V_{i}(t)=V_{i-1}(t)+v_{i}(t)+\frac{1}{2 \rho_{i}} \tilde{\theta}_{i}^{T}(t) \widetilde{\theta}_{i}(t) .
$$

The design of adaptive law is as follows:

$$
\mathscr{D}_{t}^{\beta} \theta_{i}=\rho_{i} e_{i}(t) \psi_{i}\left(\bar{x}_{i}(t)\right)-\gamma_{i} \theta_{i},
$$

where $\rho_{i}$ and $\gamma_{i}$ are positive design parameters.

Because $\theta_{i}^{*}$ is a constant, then we have

$$
\mathscr{D}_{t}^{\beta} \widetilde{\theta}_{i}=\mathscr{D}_{t}^{\beta} \theta_{i} \text {. }
$$

Then, according to Lemma 4, we can have

$$
\mathscr{D}_{t}^{\beta} V_{i}(t) \leq-k_{i-1} V_{i-1}(t)+e_{i-1}(t) e_{i}(t)+\Theta_{i-1}+\mathscr{D}_{t}^{\beta} v_{i}(t)+\frac{1}{\rho_{i}} \widetilde{\theta}_{i}^{T} \mathscr{D}_{t}^{\beta} \widetilde{\theta}_{i}
$$

From formulas (50), (52), and (54), we have

$$
\begin{aligned}
& \mathscr{D}_{t}^{\beta} V_{i}(t) \leq-k_{i-1} V_{i-1}(t)+\Theta_{i-1}+e_{i}(t) e_{i+1}(t)-k_{1 i} e_{i}^{2}-\frac{1}{\rho_{i}} \widetilde{\theta}_{i}^{T} \theta_{i} \\
& \leq-k_{i-1} V_{i-1}(t)+\Theta_{i-1}-k_{1 i} e_{i}^{2}(t)+e_{i}(t) e_{i+1}(t)-\frac{1}{\rho_{i}} \widetilde{\theta}_{i}^{T} \widetilde{\theta}_{i}-\frac{1}{\rho_{i}} \widetilde{\theta}_{i}^{T} \theta_{i}^{*} .
\end{aligned}
$$

According to Young's inequality, then we can obtain 


$$
-\frac{1}{\rho_{i}} \widetilde{\theta}_{i}^{T} \theta_{i}^{*} \leq \frac{1}{2 \rho_{i}} \theta_{i}^{* T} \theta_{i}^{*}+\frac{1}{2 \rho_{i}} \widetilde{\theta}_{i}^{T} \widetilde{\theta}_{i}
$$

Substitute the above formula into (55), the following can be obtained

$$
\begin{aligned}
\mathscr{D}_{t}^{\beta} V_{i}(t) & \leq-k_{i-1} V_{i-1}(t)+\Theta_{i-1}-k_{1 i} e_{i}^{2}(t)+e_{i}(t) e_{i+1}(t)-\frac{1}{2 \rho_{i}} \tilde{\theta}_{i}^{T} \widetilde{\theta}_{i}+\frac{1}{2 \rho_{i}} \theta_{i}^{* T} \theta_{i}^{*} \\
& \leq-k_{i} V_{i}(t)+e_{i+1}(t) e_{i}(t)+\Theta_{i},
\end{aligned}
$$

in which $k_{i}=\min \left\{k_{i-1}, 2 k_{1 i}, \gamma_{i}\right\}$ and $\Theta_{i}=\Theta_{i-1}+\left(\gamma_{i} / 2 \rho_{i}\right)$ $\theta_{i}^{* T} \theta_{i}^{*}$ are two positive constants.

Step 4. $n$.

Consider the $n$th Lyapunov function $v_{n}(x)$ as follows:

$$
v_{n}(t)=\frac{1}{2} e_{n}^{2}(t)
$$

According to Lemma 4, differentiating $v_{n}(x)$ can gain

$$
\begin{aligned}
\mathscr{D}_{t}^{\beta} v_{n}(t) \leq & e_{n}(t) \mathscr{D}_{t}^{\beta} e_{n}(t) \\
= & e_{n}(t)\left(D_{t}^{\beta} x_{n}(t)-\mathscr{D}_{t}^{\beta} x_{n, c}(t)-\mathscr{D}_{t}^{\beta} \lambda_{n}(t)\right) \\
= & e_{n}(t)\left[f_{n}\left(\bar{x}_{n}(t)\right)+d(t)+u(t)-\mathscr{D}_{t}^{\beta} x_{n, c}(t)-\mathscr{D}_{t}^{\beta} \lambda_{n}(t)\right] \\
= & e_{n}(t) f_{n}\left(\bar{x}_{n}(t)\right)-\widehat{f}_{n}\left(\bar{x}_{n}\left(t, \theta_{n}^{*}\right)\right)+\widehat{f}_{n}\left(\bar{x}_{n}\left(t, \theta_{n}^{*}\right)\right) \\
& +d(t)+u(t)-\mathscr{D}_{t}^{\beta} x_{n, c}(t)+k_{1 n} \lambda_{n}+\lambda_{n-1} \\
= & e_{n}(t)-\widetilde{\theta}_{n}^{T} \psi_{n}\left(\bar{x}_{n}(t)\right)-\epsilon_{n}\left(\bar{x}_{n}(t)\right)+\theta_{n}^{T} \psi_{n}\left(\bar{x}_{n}(t)\right) \\
& +d(t)+u(t)-\mathscr{D}_{t}^{\beta} x_{n, c}(t)+k_{1 n} \lambda_{n}+\lambda_{n-1},
\end{aligned}
$$

in which $\epsilon_{n}\left(\bar{x}_{n}(t)\right)=\hat{f}_{n}\left(\bar{x}_{n}\left(t, \theta^{*}\right)\right)-f_{n}\left(\bar{x}_{n}(t)\right)$ and $\quad$ Substituting (28) into (59), one obtains $\widetilde{\theta}_{n}=\theta_{n}-\theta_{n}^{*}$.

$$
\begin{aligned}
\mathscr{D}_{t}^{\beta} v_{n}(t) \leq & e_{n}(t)\left[-k_{1 n} e_{n}(t)-\tilde{\theta}_{n}^{T} \psi_{n}\left(\bar{x}_{n}(t)\right)-\epsilon_{n}\left(\bar{x}_{n}(t)\right)-\left(k_{2 n}+\hat{\bar{d}}(t)\right) \operatorname{sign}\left(e_{n}(t)\right)-e_{n-1}(t)+d(t)\right] \\
\leq & -k_{1 n} e_{n}^{2}(t)-e_{n}(t) \tilde{\theta}_{n}^{T} \psi_{n}\left(\bar{x}_{n}(t)\right)+\bar{\epsilon}_{n}\left|e_{n}(t)\right| \\
& -\left(k_{2 n}+\hat{\bar{d}}(t)\right)\left|e_{n}(t)\right|-e_{n-1}(t) e_{n}(t)+\bar{d}\left|e_{n}(t)\right| \\
\leq & -k_{1 n} e_{n}^{2}-e_{n}(t) \tilde{\theta}_{n}^{T} \psi_{n}\left(\bar{x}_{n}(t)\right)-e_{n-1}(t) e_{n}(t)-\tilde{\bar{d}}(t)\left|e_{n}(t)\right|,
\end{aligned}
$$

where $\underline{k}_{1 n}>0$ and $k_{2 n}>\bar{\epsilon}_{n}$ are design parameters and $\tilde{\bar{d}}(t)=$ $\bar{d}(t)-\bar{d}$ is the estimation error.

Choose the Lyapunov function $V_{n}(x)$ as follows:

$$
V_{n}(t)=V_{n-1}(t)+v_{n}(t)+\frac{1}{2 \rho_{n}} \tilde{\theta}_{n}^{T}(t) \widetilde{\theta}_{n}(t)+\frac{1}{2 \xi_{1}} \widetilde{\bar{d}}^{2}(t) .
$$

The design of adaptive law is as follows:

$$
\begin{aligned}
\mathscr{D}_{t}^{\beta} \theta_{n} & =\rho_{n} e_{n}(t) \psi_{n}\left(\bar{x}_{n}(t)\right)-\gamma_{n} \theta_{n}, \\
\mathscr{D}_{t}^{\beta} \widehat{\bar{d}}(t) & =\xi_{1}\left|e_{n}\right|-\xi_{2} \hat{\bar{d}}(t),
\end{aligned}
$$

where $\rho_{n}, \gamma_{n}, \xi_{1}$, and $\xi_{2}$ are positive design parameters.

Because $\theta_{n}^{*}$ is a constant, then we have

$$
\mathscr{D}_{t}^{\beta} \widetilde{\theta}_{n}=\mathscr{D}_{t}^{\beta} \theta_{n} .
$$

Then, according to Lemma 4, we can have 


$$
\begin{aligned}
\mathscr{D}_{t}^{\beta} V_{n}(t) \leq & -k_{n-1} V_{n-1}(t)+e_{n-1}(t) e_{n}(t)+\Theta_{n-1}+\mathscr{D}_{t}^{\beta} v_{n}(t) \\
& +\frac{1}{\rho_{n}} \widetilde{\theta}_{n}^{T} \mathscr{D}_{t}^{\beta} \widetilde{\theta}_{n}+\frac{1}{\xi_{1}} \widetilde{\bar{d}}(t) \mathscr{D}_{t}^{\beta} \widetilde{\bar{d}}(t) .
\end{aligned}
$$

$$
\begin{aligned}
\mathscr{D}_{t}^{\beta} V_{n}(t) \leq & -k_{n-1} V_{n-1}(t)+\Theta_{n-1}-k_{1 n} e_{n}^{2}-e_{n}(t) \tilde{\theta}_{n}^{T} \psi_{n}\left(\bar{x}_{n}(t)\right) \\
& -\tilde{\bar{d}}(t)\left|e_{n}(t)\right|+\frac{1}{\rho_{n}} \widetilde{\theta}_{n}^{T} \mathscr{D}_{t}^{\beta} \widetilde{\theta}_{n}+\frac{1}{\xi_{1}} \widetilde{\bar{d}}(t) \mathscr{D}_{t}^{\beta} \tilde{\overline{\bar{d}}}(t) .
\end{aligned}
$$

By substituting (62) and (63) into the above inequality, it can be obtained

$$
\begin{aligned}
\mathscr{D}_{t}^{\beta} V_{n}(t) \leq & -k_{n-1} V_{n-1}(t)+\Theta_{n-1}-k_{1 n} e_{n}^{2}-\frac{\gamma_{n}}{\rho_{n}} \tilde{\theta}_{n}^{T} \theta_{n}-\frac{\xi_{2}}{\xi_{1}} \widetilde{\bar{d}}(t) \widehat{\bar{d}}(t) \\
\leq & -k_{n-1} V_{n-1}(t)+\Theta_{n-1}-k_{1 n} e_{n}^{2}-\frac{\gamma_{n}}{\rho_{n}} \tilde{\theta}_{n}^{T} \widetilde{\theta}_{n}-\frac{\gamma_{n}}{\rho_{n}} \tilde{\theta}_{n}^{T} \theta_{n}^{*} \\
& -\frac{\xi_{2} \widetilde{\bar{d}}^{2}}{\xi_{1}}(t)-\frac{\xi_{2}}{\xi_{1}} \widetilde{\bar{d}}(t) \bar{d} .
\end{aligned}
$$

According to Young's inequality, we can obtain

$$
\begin{aligned}
& -\frac{1}{\rho_{i}} \widetilde{\theta}_{n}^{T} \theta_{n}^{*} \leq \frac{1}{2 \rho_{n}} \theta_{n}^{* T} \theta_{n}^{*}+\frac{1}{2 \rho_{n}} \widetilde{\theta}_{n}^{T} \widetilde{\theta}_{n}, \\
& -\frac{\xi_{2}}{\xi_{1}} \widetilde{\bar{d}}(t) \bar{d}(t) \leq \frac{\xi_{2}}{2 \xi_{1}} \widetilde{\bar{d}}^{2}(t)+\frac{\xi_{2}}{2 \xi_{1}} \bar{d}^{2} .
\end{aligned}
$$

$$
\begin{aligned}
\mathscr{D}_{t}^{\beta} V_{i}(t) \leq & -k_{n-1} V_{n-1}(t)+\Theta_{n-1}-k_{1 n} e_{n}^{2}-\frac{\gamma_{n}}{2 \rho_{n}} \widetilde{\theta}_{n}^{T} \widetilde{\theta}_{n}+\frac{\gamma_{n}}{2 \rho_{n}} \theta_{n}^{* T} \theta_{n}^{*} \\
& -\frac{\xi_{2}}{2 \xi_{1}} \widetilde{\bar{d}}^{2}(t)+\frac{\xi_{2}}{2 \xi_{1}} \bar{d}^{2} \leq-k_{n} V_{n}(t)+\Theta_{n},
\end{aligned}
$$

in which $k_{n}=\min \left\{k_{\underline{n}-1}, 2 k_{1 n}, \gamma_{n}, \xi_{2}\right\}$ and $\Theta_{n}=\Theta_{n-1}+\left(\gamma_{n} /\right.$ $\left.2 \rho_{n}\right) \theta_{n}^{* T} \theta_{n}^{*}+\left(\xi_{2} / 2 \xi_{1}\right) d^{2}$ are two positive constants.

The stability analysis of fractional-order nonlinear systems is shown below:

Theorem 1. Consider system (20) under Assumption 1. The control input is constructed as (29) with (26)-(28), and the adaptation laws are designed as (34), (44), (52), (62), and (63); then, there exist appropriate design parameters, such that the tracking error $e_{1}(t)$ tends to an arbitrarily small region of the origin.

Proof. According to (70), there exists a function $q(t) \geq 0$ such that the following equation holds on:

$$
\mathscr{D}_{t}^{\beta} V_{i}(t)+q(t)=-k_{n} V_{n}(t)+\Theta_{n} .
$$
obtain

The Laplace transform of equation (71) is applied to 


$$
\begin{aligned}
V_{n}(s) & =\frac{s^{\beta-1}}{s^{\beta}+k_{n}} V_{n}(0)+\frac{\Theta_{n}}{s\left(s^{\beta}+k_{n}\right)}-\frac{Q(s)}{s^{\beta}+k_{n}} \\
& =\frac{s^{\beta-1}}{s^{\beta}+k_{n}} V_{n}(0)+\frac{s^{\beta-(1+\beta)} \Theta_{n}}{s_{\beta}+k_{n}}-\frac{Q(s)}{s^{\beta}+k_{n}},
\end{aligned}
$$

in which $V_{n}(s)$ and $Q(s)$ are Laplace transforms of $V_{n}(t)$ and $q(t)$, respectively, and $V_{n}(0)$ is the initial condition. According to (6) and (71), it can be represented as

$$
V_{n}(t)=V_{n}(0) E_{\beta, 1}\left(-k_{n} t^{\beta}\right)+\Theta_{n} t^{\beta} E_{\beta, 1+\beta}\left(-k_{n} t^{\beta}\right)-q(t) * t^{-1} E_{\beta, 0}\left(-k_{n} t^{\beta}\right),
$$

where $*$ is the convolution operator. If sine $q(t)$ and $t^{-1} E_{\beta, 0}\left(-k_{n} t^{\beta}\right)$ are both nonnegative functions, then $q(t) * t^{-1} E_{\beta, 0}\left(-k_{n} t^{\beta}\right) \geq 0$ can be obtained. Hence, the following inequality holds on:

$$
\left|V_{n}(t)\right| \leq\left|V_{n}(0)\right| E_{\beta, 1}\left(-k_{n} t^{\beta}\right)+\Theta_{n} t^{\beta} E_{\beta, 1+\beta}\left(-k_{n} t^{\beta}\right) .
$$

Because $\arg \left(-k_{n} t^{\beta}\right)=-\pi,\left|-k_{n} t^{\beta}\right| \geq 0$, for all $t \geq 0$ and $\beta \in(0,2)$ and according to Lemma 2 , then there exists a positive constant $A$ such that

$$
\left|E_{\beta, 1}\left(-k_{n} t^{\beta}\right)\right| \leq \frac{A}{1+k_{n} t^{\beta}} .
$$

According to (75), one can obtain

$$
\lim _{t \rightarrow \infty}\left|V_{n}(0)\right| E_{\beta, 1}\left(-k_{n} t^{\beta}\right)=0
$$

Consequently, for every $\eta>0$, there exists a constant $t_{r}>0$, such that $t>t_{r}$ holds on, then one can obtain

$$
\left|V_{n}(0)\right| E_{\beta, 1}\left(-k_{n} t^{\beta}\right)<\frac{\eta}{3} \text {. }
$$

In addition, according to Lemma 1 , one can obtain

$$
E_{\beta, \beta+1}\left(-k_{n} t^{\beta}\right)=\frac{1}{\Gamma(1) k_{n} t^{\beta}}+o\left(\frac{1}{\left|-k_{n} t^{\beta}\right|^{1+1}}\right),
$$

in which the integer $n$ is chosen as 1 . According to (78), for every $\eta>0$, there exists a constant $t_{b}>0$, such that $t>t_{b}$ holds on, one obtains

$$
\Theta_{n} t^{\eta} E_{\eta, \eta+1}\left(-k_{n} t^{\eta}\right) \leq \frac{\Theta_{n}}{k_{n}}+\frac{\eta}{3}
$$

Then, we can select appropriate design parameters such that $\left(\Theta_{n} / n\right) \leq(\eta / 3)$. Therefore, from (74), (77), and (79), the following inequality can be obtained:

$$
\left|V_{n}(t)\right|<\eta \text {. }
$$

According to (80) and the definition of $V_{n}(t)$, all signals in the closed-loop system are bounded and the tracking error $e_{1}(t)$ tends to an arbitrary small region of the origin, i.e., for all $t>\max \left\{t_{r}, t_{b}\right\}$, such that $(1 / 2) e_{1}^{2}(t) \leq \eta$.

\section{Simulation Results}

In this section, two examples will be given to demonstrate the effectiveness of the adaptive neural network backstepping control based on the command filtering.

Example 1. The fractional-order Duffing's Oscillator chaos system is as follows:

$$
\left\{\begin{array}{l}
\mathscr{D}_{t}^{\beta} x_{1}(t)=x_{2}(t), \\
\mathscr{D}_{t}^{\beta} x_{2}(t)=x_{1}(t)-x_{1}^{3}(t)-0.5 x_{2}(t)+1.3 \cos t+d(t)+u(t),
\end{array}\right.
$$

in which $f_{1}\left(x_{1}(t)\right)=0$ and $f_{2}\left(x_{1}(t), x_{2}(t)\right)=x_{1}$ $(t)-x_{1}^{3}(t)-0.5 x_{2}(t)+1.3 \cos t$ are unknown functions. Let the fractional-order $\beta=0.95$ and the initial conditions $x_{1}=0.21$ and $x_{2}=0.13$. In addition, it can be seen from Figures 1 and 2 that the nonlinear system is unstable when both the controller $u(t)$ and the disturbance signal $d(t)$ of the system are zero.

The known smooth reference signal $x_{d}(t)$ and the unknown external disturbance signal $d(t)$ are chosen as $\sin (t)$ and $\sin (t)+\cos (t)$, respectively. The parameters to be designed are selected as follows: $k_{11}=k_{12}=1, k_{21}=k_{22}=1$, $\rho_{1}=\rho_{2}=5, \gamma_{1}=\gamma_{2}=0.5, \xi_{1}=\xi_{2}=1$, and $\kappa_{1}=50$. In the process of designing the controller, $\operatorname{sign}(\cdot)$ is used to represent $\arctan (10)$ to avoid the chattering phenomenon.

Next, let us design the radial-basis-functions. According to the property of the radial-basis-function $\mathrm{NN}$, the input variable is $x_{1}(t)$ in the first radial-basis-function and six Gaussian functions evenly distributed within the interval $[-5,5]$ are designed. The second radial-basis-function uses $x_{1}(t)$ and $x_{2}(t)$ as input variables. Same as the first radialbasis-function, each input variable corresponds to six Gaussian functions evenly distributed on $[-5,5]$. The initial conditions are presented to be $\theta_{1}(0)=[1,1,1,1,1,1]^{T} \in \mathscr{R}^{6}$ and $\theta_{2}(0)=[1,1, \ldots, 1]^{T} \in \mathscr{R}^{36}$. 


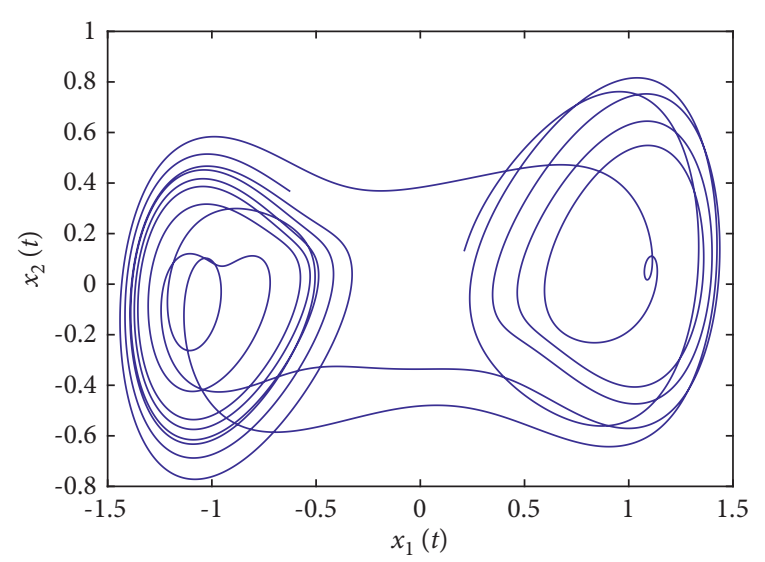

Figure 1: The uncontrolled fractional-order Duffing's oscillator chaos system (81).

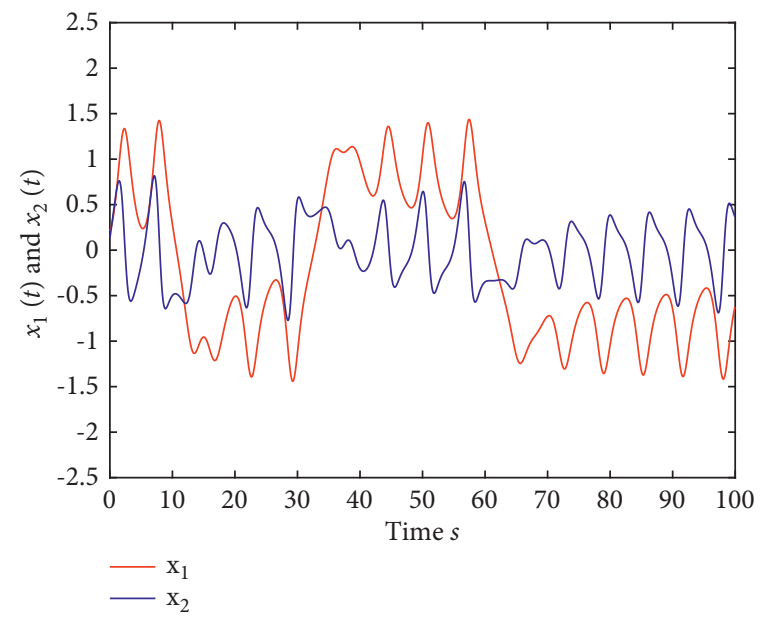

FIgURE 2: The time response of the state variable of (81) when $u(t)=0$ and $d(t)=0$.

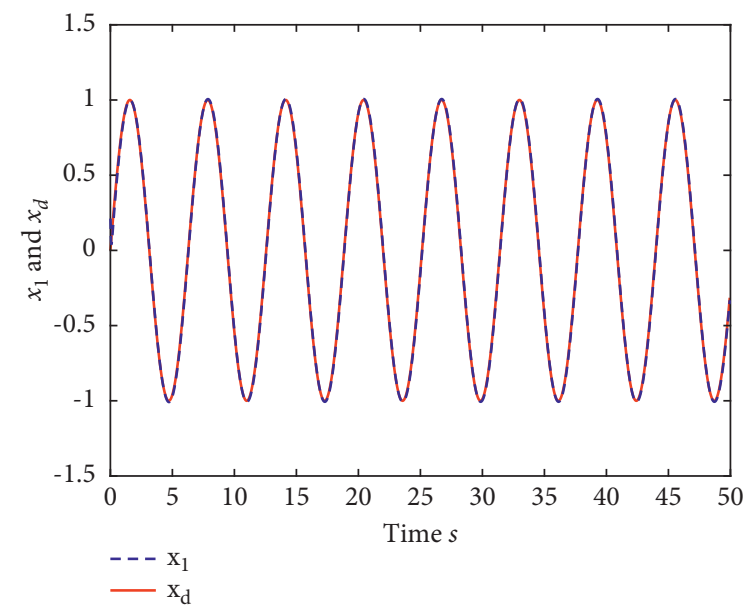

Figure 3: The trace of $x_{1}$ and $x_{d}$ of system (81).

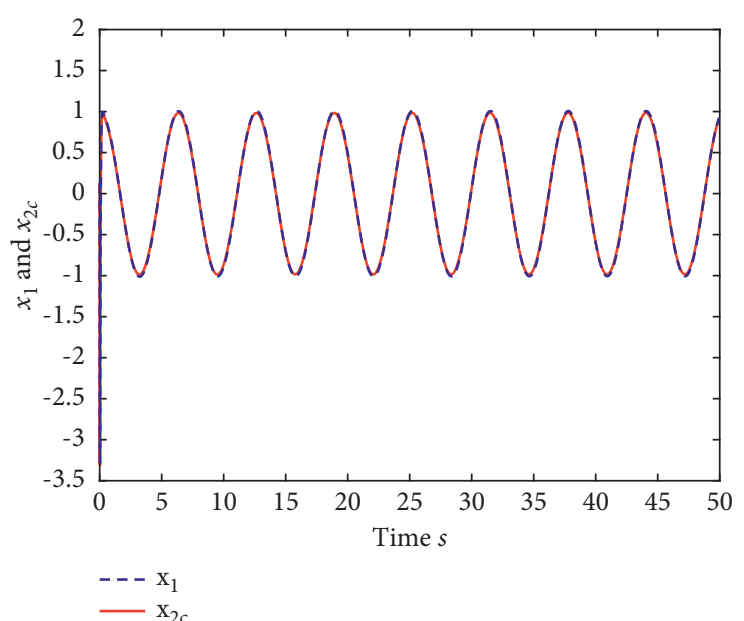

Figure 4: The trace of $x_{2}$ and $x_{2 c}$ of system (81).

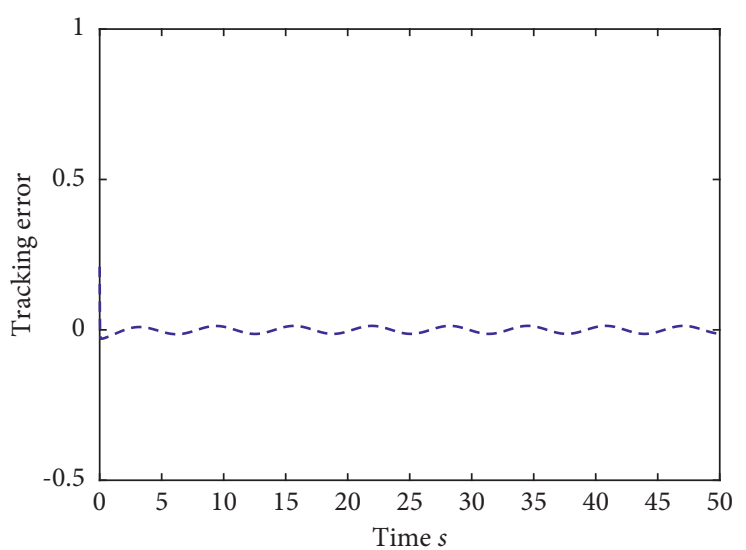

Figure 5: The tracking error of the output $y(t)$ of system (81).

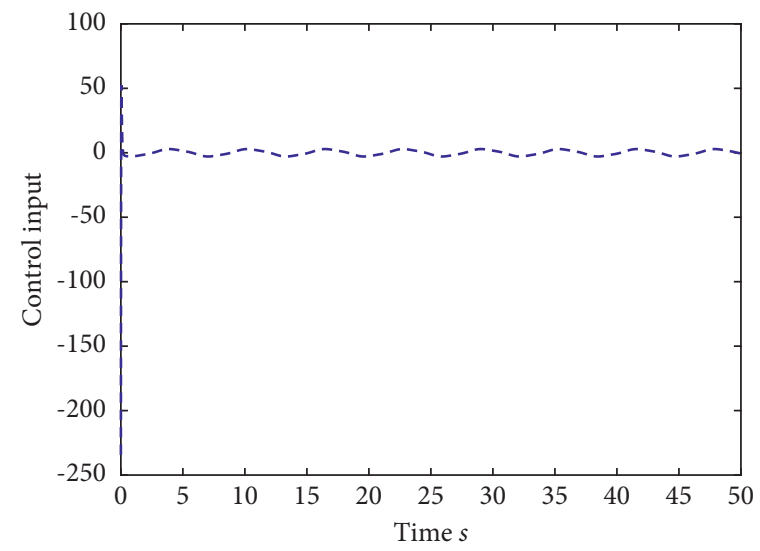

FIgure 6: The control input of system (81). 


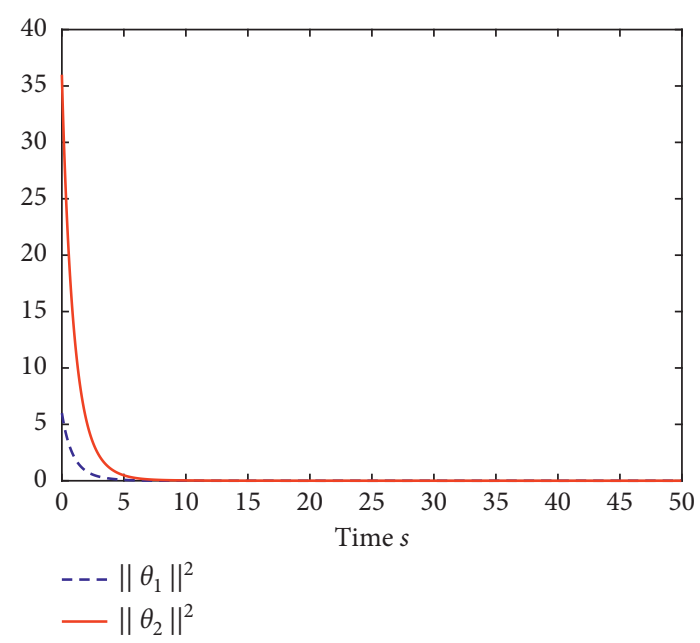

FIgURE 7: The adaptive laws of system (81).

When the above initial conditions are met, the simulation of the nonlinear system (81) is shown in Figures 3-7.

As can be seen from Figures 3 and 4, the state variables $x_{1}(t)$ and $x_{2}(t)$ of the system are tracked by signals $x_{d}(t)$ and $x_{2 c}(t)$ and the tracking effect is relatively well. As shown in Figure 5, the tracking error $e_{1}(t)$ rapidly converges to a relatively small neighborhood of the origin. From Figure 6, the control input is large at first, then decreases rapidly, and when the system reaches stability, it is controlled in a relatively small neighborhood. As shown in Figure 7, the adaptive laws of the system are bounded and converge rapidly to the neighborhood of the origin. Consequently, the effectiveness of the adaptive neural network backstepping control method based on command filtering for a class of classical fractional-order nonlinear strictly feedback systems.

Example 2. The fractional-order Arneodo's chaos system is as follows:

$$
\left\{\begin{array}{l}
\mathscr{D}_{t}^{\beta} x_{1}(t)=x_{2}(t), \\
\mathscr{D}_{t}^{\beta} x_{2}(t)=x_{3}(t), \\
\mathscr{D}_{t}^{\beta} x_{2}(t)=5.5 x_{1}(t)-3.5 x_{2}(t)-0.8 x_{3}(t)+x_{1}^{3}(t)+d(t)+u(t),
\end{array}\right.
$$

where $\beta=0.97, x_{1}(0)=-0.2, x_{2}(0)=0.5$, and $x_{3}(0)=0.2$. If the input and disturbance of the system satisfy $u(t)=0$ and $d(t)=0$, the state variables of the system are shown in Figures 8 and 9.

The known smooth reference signal $x_{d}(t)$ and the unknown external disturbance signal $d(t)$ are $\sin (t)$ and $2 \sin (t) \cos (t)$, respectively. The parameters to be designed are selected as follows: $k_{11}=40, k_{12}=25, \quad k_{13}=30$, $k_{21}=k_{22}=k_{23}=15, \rho_{1}=\rho_{2}=\rho_{2}=15, \gamma_{1}=\gamma_{2}=\gamma_{3}=0.5$, $\xi_{1}=\xi_{2}=1, \kappa_{1}=1$, and $\kappa_{2}=20$.

In Example 2, there are three radial-basis-function neural networks. In the first radial-basis-function $\mathrm{NN}$, the input variable is $x_{1}(t)$ and four Gaussian functions evenly distributed within the interval $[-3,3]$ are designed. The second radial-basis-function uses $x_{1}(t)$ and $x_{2}(t)$ as input

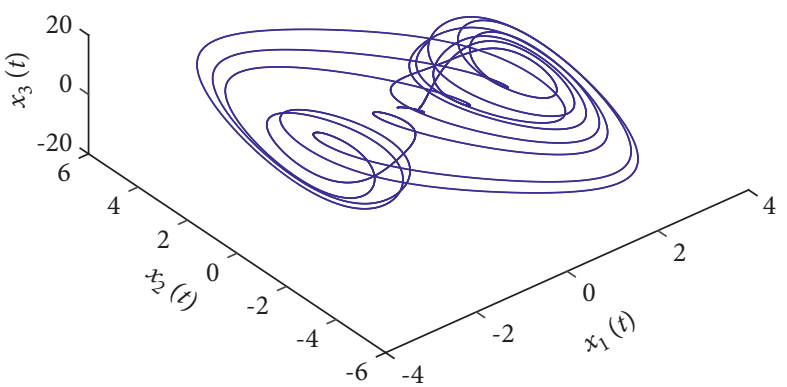

Figure 8: The uncontrolled fractional-order Arneodo's chaos system (81).

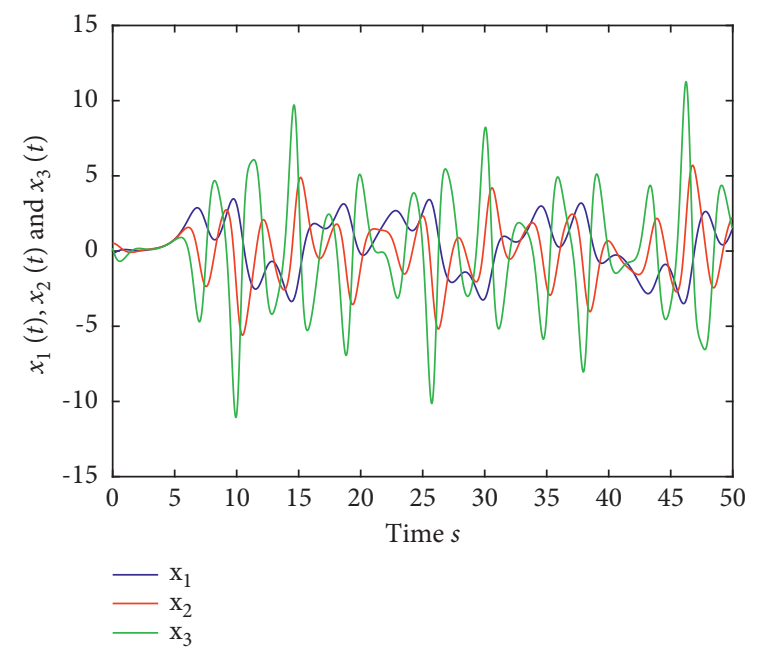

Figure 9: The time response of the state variable of (81) when $u(t)=0$ and $d(t)=0$.

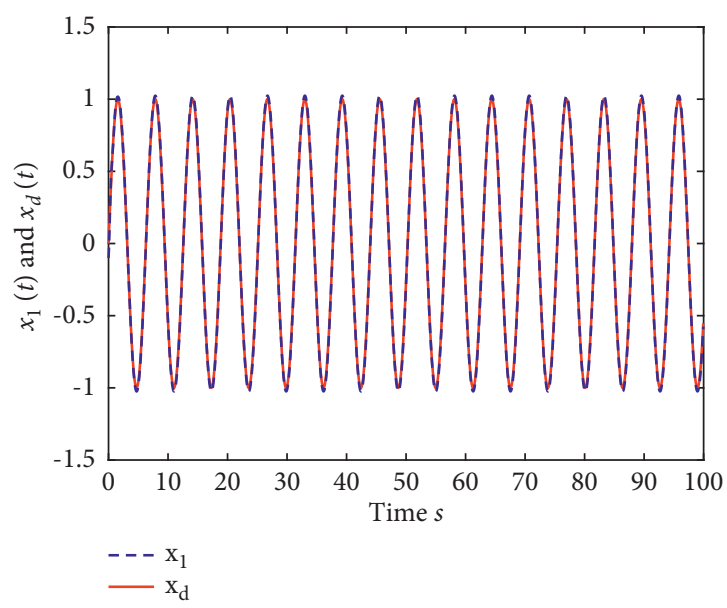

Figure 10: The trace of $x_{1}$ and $x_{d}$ of system (81).

variables. Same as the first radial-basis-function, each input variable corresponds to four Gaussian functions evenly distributed on $[-3,3]$. The third radial-basis-function uses $x_{1}(t), x_{2}(t)$, and $x_{3}(t)$ as input variables. Same as the before radial-basis-functions, four Gaussian functions in every 


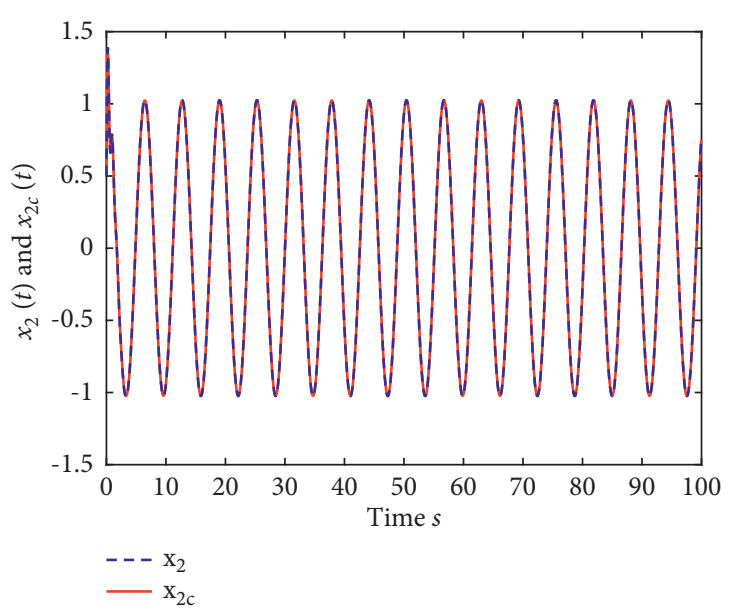

Figure 11: The trace of $x_{2}$ and $x_{2 c}$ of system (81).

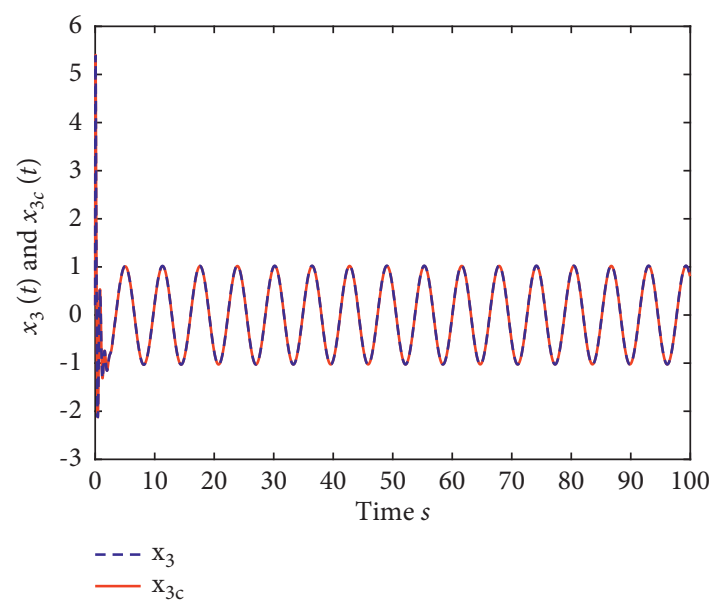

Figure 12: The trace of $x_{3}$ and $x_{3 c}$ of system (81).

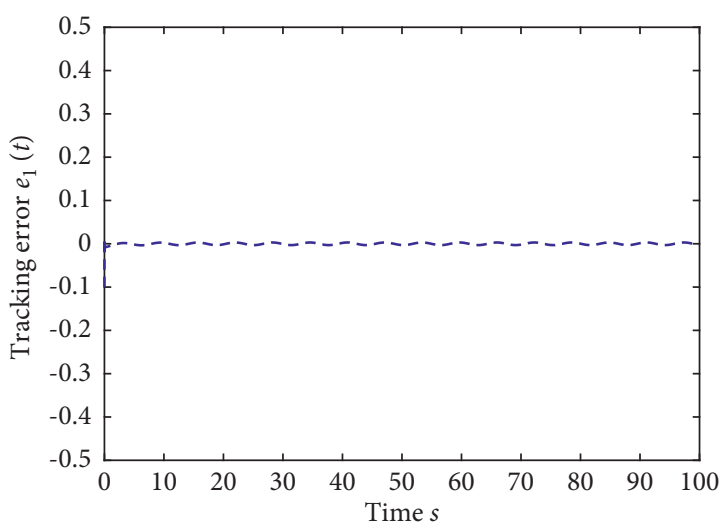

FIgURE 13: The tracking error of the output $y(t)$ of system (81).

input variable are evenly distributed on $[-3,3]$. The initial conditions are presented to be $\theta_{1}(0)=[1,1,1,1]^{T} \in \mathscr{R}^{4}$, $\theta_{2}(0)=[1,1, \ldots, 1]^{T} \in \mathscr{R}^{16}$, and $\theta_{3}(0)=[1,1, \ldots, 1]^{T}$

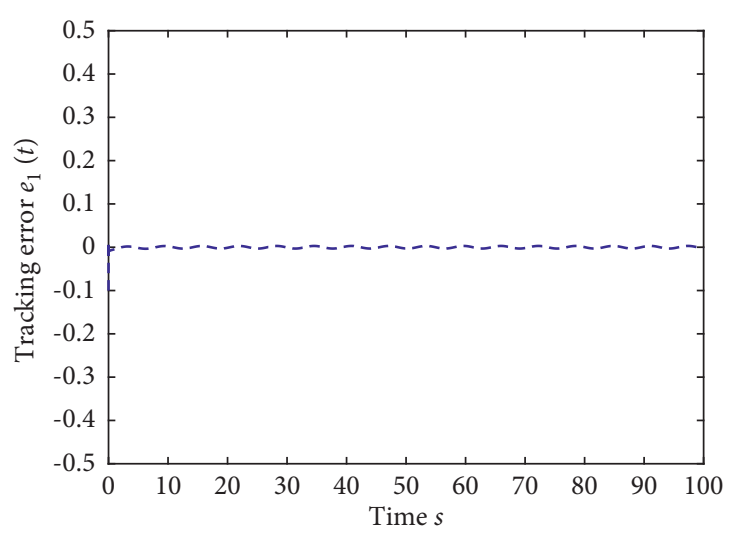

FIgURE 14: The control input of system (81).

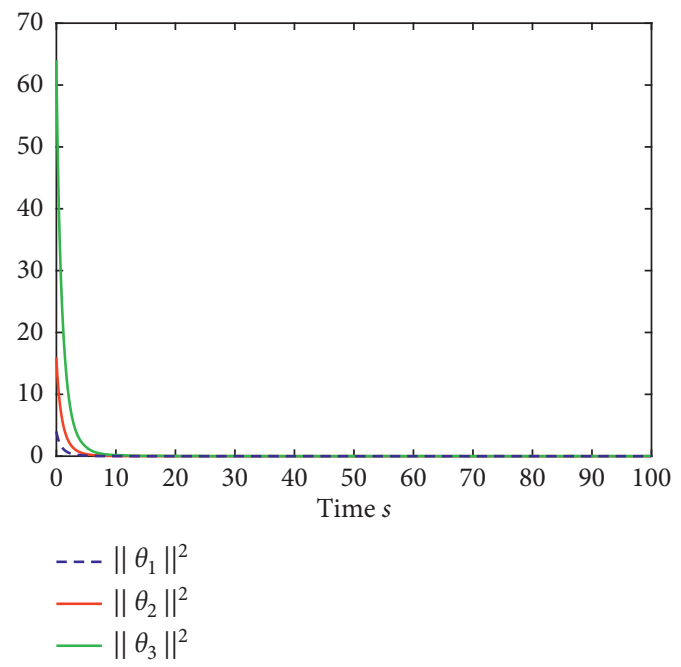

Figure 15: The adaptive laws of system (81).

$\in \mathscr{R}^{64}$. When the above initial conditions are met, the simulation of the nonlinear system (81) is shown in Figures 10-15.

The simulation results of Example 2 are shown above, which is similar to the result analysis of Example 1, and the final results are the same as that of Example 1.

\section{Conclusion}

In this paper, an adaptive neural network backstepping control method based on command filtering is proposed for a class of fractional-order strictly feedback nonlinear systems. The command filtering technology is used to deal with the explosive of terms in the traditional backstepping technology, and the error compensation mechanism is introduced to overcome the shortcomings of the dynamic surface method. The simulation results of the fractionalorder nonlinear strictly feedback system show the effectiveness of the proposed method. The future research will be adaptive neural network control of fractional-order nonlinear strictly feedback system with disturbance observer based on command filtering. 


\section{Data Availability}

All the datasets generated for this study are included within the article.

\section{Conflicts of Interest}

The author declares that there are no conflicts of interest regarding the publication of this paper.

\section{References}

[1] J. Shen and J. Lam, "Non-existence of finite-time stable equilibria in fractional-order nonlinear systems," Automatica, vol. 50, no. 2, pp. 547-551, 2014.

[2] N. Aguila-Camacho, M. A. Duarte-Mermoud, and J. A. Gallegos, "Lyapunov functions for fractional order systems," Communications in Nonlinear Science and $\mathrm{Nu}$ merical Simulation, vol. 19, no. 9, pp. 2951-2957, 2014.

[3] H. Liu, Y. Pan, and J. Cao, "Composite learning adaptive dynamic surface control of fractional-order nonlinear systems," IEEE Transactions on Cybernetics, vol. 50, no. 6, pp. 2557-2567, 2020.

[4] H.-B. Bao and J.-D. Cao, "Projective synchronization of fractional-order memristor-based neural networks," Neural Networks, vol. 63, pp. 1-9, 2015.

[5] H. Liu, Y. Pan, S. Li, and Y. Chen, "Adaptive fuzzy backstepping control of fractional-order nonlinear systems," IEEE Transactions on Systems, Man, and Cybernetics: Systems, vol. 47, no. 8, pp. 2209-2217, 2017.

[6] L. Heng, L. Sheng-Gang, S. Ye-Guo, and W. Hong-Xing, "Prescribed performance synchronization for fractional-order chaotic systems," Chinese Physics B, vol. 24, no. 9, Article ID 090505, 2015.

[7] Y. Li, Y. Chen, and I. Podlubny, "Mittag-leffler stability of fractional order nonlinear dynamic systems," Automatica, vol. 45, no. 8, pp. 1965-1969, 2009.

[8] E. A. Boroujeni and H. R. Momeni, "Non-fragile nonlinear fractional order observer design for a class of nonlinear fractional order systems," Signal Processing, vol. 92, no. 10, pp. 2365-2370, 2012.

[9] Y. Yang and G. Chen, "Finite-time stability of fractional order impulsive switched systems," International Journal of Robust and Nonlinear Control, vol. 25, no. 13, pp. 2207-2222, 2015.

[10] H. Liu, S. Li, H. Wang, Y. Huo, and J. Luo, "Adaptive synchronization for a class of uncertain fractional-order neural networks," Entropy, vol. 17, no. 10, pp. 7185-7200, 2015.

[11] X. Huang, Z. Wang, Y. Li, and J. Lu, "Design of fuzzy state feedback controller for robust stabilization of uncertain fractional-order chaotic systems," Journal of the Franklin Institute, vol. 351, no. 12, pp. 5480-5493, 2014.

[12] X. Liu, G. Gu, and K. Zhou, "Robust stabilization of mimo nonlinear systems by backstepping," Automatica, vol. 35, no. 5, pp. 987-992, 1999.

[13] M. Krstic, P. V. Kokotovic, and I. Kanellakopoulos, Nonlinear and Adaptive Control Design, John Wiley and Sons, Hoboken, NJ, USA, 1995.

[14] I. Kanellakopoulos, P. V. Kokotovic, and A. S. Morse, "Systematic design of adaptive controllers for feedback linearizable systems," IEEE Transactions on Automatic Control, vol. 36, pp. 649-654, 1991.

[15] W. Chen, L. Jiao, J. Li, and R. Li, “Adaptive nn backstepping output-feedback control for stochastic nonlinear strict-feedback systems with time-varying delays," IEEE Transactions on
Systems, Man, and Cybernetics, Part B (Cybernetics), vol. 40, no. 3, pp. 939-950, 2009.

[16] A. Boulkroune, M. Farza, and M. M'Saad, "Fuzzy approximation-based indirect adaptive controller for multi-input multi-output non-affine systems with unknown control direction," IET Control Theory and Applications, vol. 6, no. 17, pp. 2619-2629, 2012.

[17] L. Heng, Y. Hai-Jun, and X. Wei, "Adaptive fuzzy nonlinear inversion-based control for uncertain chaotic systems," Chinese Physics B, vol. 21, no. 12, Article ID 120505, 2012.

[18] T.-S. Li, S.-C. Tong, and G. Feng, "A novel robust adaptivefuzzy-tracking control for a class of nonlinear multi-input/ multi-output systems," IEEE Transactions on Fuzzy Systems, vol. 18, no. 1, pp. 150-160, 2009.

[19] T.-S. Tie-Shan Li, D. Dan Wang, G. Gang Feng, and S.-C. Shao-Cheng Tong, "A DSC approach to robust adaptive NN tracking control for strict-feedback nonlinear systems," IEEE Transactions on Systems, Man, and Cybernetics, Part B (Cybernetics), vol. 40, no. 3, pp. 915-927, 2010.

[20] Y. Li, S. Tong, and T. Li, "Hybrid fuzzy adaptive output feedback control design for uncertain mimo nonlinear systems with time-varying delays and input saturation," IEEE Transactions on Fuzzy Systems, vol. 24, no. 4, pp. 841-853, 2015.

[21] S.-C. Shao-Cheng Tong, X.-L. Xiang-Lei He, and H.-G. HuaGuang Zhang, "A combined backstepping and small-gain approach to robust adaptive fuzzy output feedback control," IEEE Transactions on Fuzzy Systems, vol. 17, no. 5, pp. 1059-1069, 2009.

[22] W. Dong, J. A. Farrell, M. M. Polycarpou, V. Djapic, and M. Sharma, "Command filtered adaptive backstepping," IEEE Transactions on Control Systems Technology, vol. 20, no. 3, pp. 566-580, 2011.

[23] J. Hu and H. Zhang, "Immersion and invariance based command-filtered adaptive backstepping control of vtol vehicles," Automatica, vol. 49, no. 7, pp. 2160-2167, 2013.

[24] W. Assawinchaichote and S. K. Sing Kiong Nguang, "Fuzzy $\mathrm{H} / \mathrm{sub} / \mathrm{spl}$ infin//output feedback control design for singularly perturbed systems with pole placement constraints: an LMI approach," IEEE Transactions on Fuzzy Systems, vol. 14, no. 3, pp. 361-371, 2006.

[25] Q. Shen, B. Jiang, and V. Cocquempot, "Adaptive fuzzy observer-based active fault-tolerant dynamic surface control for a class of nonlinear systems with actuator faults," IEEE Transactions on Fuzzy Systems, vol. 22, no. 2, pp. 338-349, 2013.

[26] Q. Shen, B. Jiang, and V. Cocquempot, "Fuzzy logic systembased adaptive fault-tolerant control for near-space vehicle attitude dynamics with actuator faults," IEEE Transactions on Fuzzy Systems, vol. 21, no. 2, pp. 289-300, 2012.

[27] M. Chadli and H. R. Karimi, "Robust observer design for unknown inputs takagi-sugeno models," IEEE Transactions on Fuzzy Systems, vol. 21, no. 1, pp. 158-164, 2012.

[28] M. Chadli and T. M. Guerra, "LMI solution for robust static output feedback control of discrete takagi-sugeno fuzzy models," IEEE Transactions on Fuzzy Systems, vol. 20, no. 6, pp. 1160-1165, 2012.

[29] J. Yu, P. Shi, W. Dong, B. Chen, and C. Lin, "Neural networkbased adaptive dynamic surface control for permanent magnet synchronous motors," IEEE Transactions on Neural Networks and Learning Systems, vol. 26, no. 3, pp. 640-645, 2014.

[30] J. Yu, P. Shi, H. Yu, B. Chen, and C. Lin, “Approximationbased discrete-time adaptive position tracking control for 
interior permanent magnet synchronous motors," IEEE Transactions on Cybernetics, vol. 45, no. 7, pp. 1363-1371, 2014.

[31] J. Wang, "Adaptive fuzzy control of direct-current motor dead-zone systems," International Journal of Innovative Computing, Information and Control, vol. 10, no. 4, pp. 1391-1399, 2014.

[32] M. Chen and S. S. Ge, "Direct adaptive neural control for a class of uncertain nonaffine nonlinear systems based on disturbance observer," IEEE Transactions on Cybernetics, vol. 43, no. 4, pp. 1213-1225, 2012.

[33] J. Yu, Y. Ma, H. Yu, and C. Lin, "Adaptive fuzzy dynamic surface control for induction motors with iron losses in electric vehicle drive systems via backstepping," Information Sciences, vol. 376, pp. 172-189, 2017.

[34] Y. Li, S. Tong, and T. Li, "Composite adaptive fuzzy output feedback control design for uncertain nonlinear strict-feedback systems with input saturation," IEEE Transactions on Cybernetics, vol. 45, no. 10, pp. 2299-2308, 2014.

[35] Y.-J. Liu, S. Tong, D.-J. Li, and Y. Gao, "Fuzzy adaptive control with state observer for a class of nonlinear discrete-time systems with input constraint," IEEE Transactions on Fuzzy Systems, vol. 24, no. 5, pp. 1147-1158, 2015.

[36] B. Xu, F. Sun, Y. Pan, and B. Chen, "Disturbance observer based composite learning fuzzy control of nonlinear systems with unknown dead zone," IEEE Transactions on Systems, Man, and Cybernetics: Systems, vol. 47, no. 8, pp. 1854-1862, 2016.

[37] D. Baleanu, J. A. T. Machado, and A. C. Luo, Fractional Dynamics and Control, Springer Science and Business Media, Berlin, Germany, 2011.

[38] D. Ding, D. Qi, Y. Meng, and L. Xu, “Adaptive mittag-leffler stabilization of commensurate fractional-order nonlinear systems," in Proceedings of the 53rd IEEE Conference on Decision and Control, pp. 6920-6926, Los Angeles, CA, USA, December 2014.

[39] Y. Wei, Y. Chen, S. Liang, and Y. Wang, "A novel algorithm on adaptive backstepping control of fractional order systems," Neurocomputing, vol. 165, pp. 395-402, 2015. 